\title{
Control of dynamic stability of stays using passive viscous dampers
}

Pietro Croce, Paolo Formichi, Filippo Landi, Roberto Castelluccio

\begin{abstract}
Since cable vibrations cause cable-stayed bridge users discomfort and may also lead to bridge collapse, assessment and control of dynamic behavior of stays are key aspects in designing such a bridge type. Mounting viscous dampers close to deck anchorages is an efficient way to control all kind of cable vibrations. In the paper, relevant issues as parametric excitation, external excitation and cable-structure interaction are investigated in order to define the required damping ratio to control the dynamic stability of stays. The study ends with a damper design example, referring to a relevant case study.
\end{abstract}

Keywords - cable-stayed bridge, cable vibrations, cablestructure interaction, viscous damper.

\section{Introduction}

Assessment and control of dynamic behavior of stays are key aspects in designing cable stayed bridges, as demonstrated by several vibration episodes occurred in all over the world as those described in [1]. Moreover, since the stays are very flexible elements, with small damping coefficients $(\xi \leq 0.50 \%)$, they are very vulnerable to vibrations induced by dynamic actions.

Aim of this paper is to investigate the dynamic stability of stays under indirect excitation and to define a possible countermeasure for its improvement.

Considering that mounting viscous dampers close to deck anchorages is an efficient passive measure to control all kind of cable vibrations, this control technique is discussed and the design of a suitable viscous damper is illustrated referring to a relevant cases study.

\section{Cable vibration phenomena}

In cable stayed bridges, dynamic actions induced by wind, traffic and earthquakes produce also an indirect excitation of cables through the motion of their anchorages. Generally two kind of excitations are distinguished: external and parametric. The former corresponds to a motion of anchorages perpendicular to the cable chord, the latter corresponds to a motion of anchorages in the direction of cable chord. Designers also have to verify cable-structure interaction: if some cable frequencies are close to bridge global frequencies, an internal resonance could occur.

Pietro Croce, Paolo Formichi, Filippo Landi, Roberto Castelluccio University of Pisa, Department of Civil and Industrial Engineering Structural Division Italy

\section{The case study}

The considered case study refers to a cable-stayed bridge designed to cross the river Arno in Figline Valdarno, a town near Florence [2]. This self-anchored cable-stayed bridge has a total length of $281 \mathrm{~m}$ with three spans respectively of $44 \mathrm{~m}, 37 \mathrm{~m}$ and $200 \mathrm{~m}$ (Fig. 1). The $\mathrm{S} 355$ steel box girder is trapezoidal, $2.00 \mathrm{~m}$ in depth and $14.96 \mathrm{~m}$ width (Fig. 2), and the $12 \mathrm{~mm}$ thick orthotropic steel deck plate is reinforced with trapezoidal stiffeners spaced $600 \mathrm{~mm}$ center to center.

The deck is suspended to a pylon via 17 pairs of lockedcoil strands forming an asymmetric semi-fan; these cables are $15.0 \mathrm{~m}$ spaced on the girder and $3.75 \mathrm{~m}$ on the pylon. The concrete A-shaped pylon has a total height of $96.00 \mathrm{~m}$. All substructures are founded on piles with a diameter of $1.60 \mathrm{~m}$.

Structural analysis has been carried out with SAP $2000^{\circledR}$.

\section{Case analysis}

\section{A. Natural frequencies of cables}

Natural frequencies of cables have been evaluated with Mehrabi and Tabatabai formula [3] as reported in a companion paper [4]. Table I summarizes these results, together with cable inclination $(\theta)$, cable chord length $(L)$, cable sag at midpoint $(f)$, cable diameter $(D)$ and cable tension at the dead load configuration $(T)$. These cables are sensibly stretched, so in the analysis an equivalent stiffness has been taken into account through the Irvine parameter [5], without resorting to more refined theoretical models [6].

\begin{tabular}{|c|c|c|c|c|c|c|}
\hline Stay & $\begin{array}{c}\theta \\
\langle\bar{\gamma}\end{array}$ & $\begin{array}{c}L \\
(\mathrm{~m})\end{array}$ & $\underset{(\mathrm{m})}{f}$ & $\begin{array}{c}D \\
(\mathrm{~mm})\end{array}$ & $\begin{array}{c}T \\
(\mathrm{kN})\end{array}$ & $\begin{array}{c}f_{1} \\
\left(\mathrm{~Hz}_{2}\right)\end{array}$ \\
\hline 1 & 48 & 122 & 0.750 & 230 & 7335 & 0.717 \\
\hline 2 & 51 & 106 & 0.247 & 55 & 942 & 1.129 \\
\hline 3 & 54 & 88 & 0.198 & 55 & 798 & 1.264 \\
\hline 4 & 58 & 69 & 0.172 & 230 & 10318 & 1.459 \\
\hline 5 & 66 & 52 & 0.069 & 60 & 964 & 2.181 \\
\hline 6 & 65 & 51 & 0.078 & 65 & 965 & 2.059 \\
\hline 7 & 54 & 62 & 0.156 & 65 & 707 & 1.451 \\
\hline 8 & 46 & 74 & 0.193 & 65 & 821 & 1.291 \\
\hline 9 & 40 & 88 & 0.264 & 70 & 971 & 1.106 \\
\hline 10 & 37 & 102 & 0.325 & 70 & 1062 & 0.995 \\
\hline 11 & 34 & 116 & 0.401 & 70 & 1123 & 0.895 \\
\hline 12 & 31 & 131 & 0.493 & 75 & 1334 & 0.809 \\
\hline 13 & 30 & 146 & 0.589 & 75 & 1383 & 0.740 \\
\hline 14 & 28 & 161 & 0.694 & 75 & 1428 & 0.683 \\
\hline 15 & 27 & 176 & 0.791 & 75 & 1500 & 0.640 \\
\hline 16 & 26 & 191 & 0.935 & 75 & 1499 & 0.591 \\
\hline 17 & 25 & 206 & 0.872 & 70 & 1628 & 0.605 \\
\hline
\end{tabular}




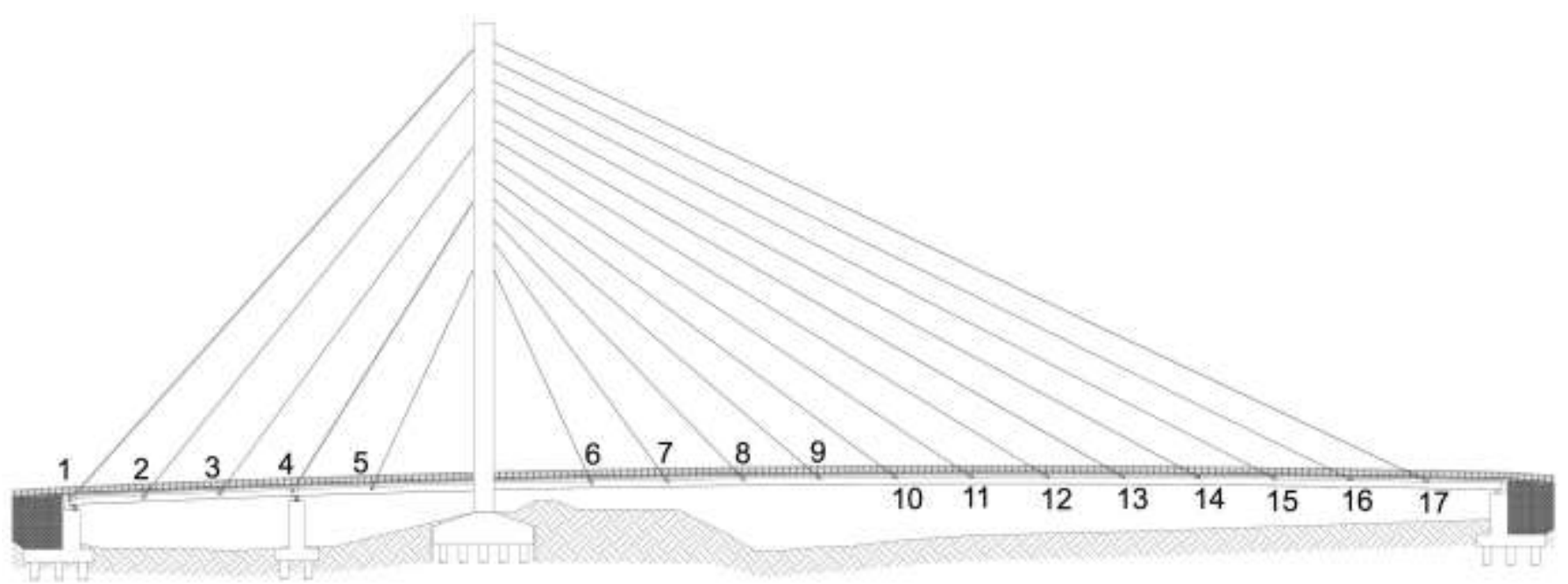

Figure 1. The cable-stayed bridge analyzed.

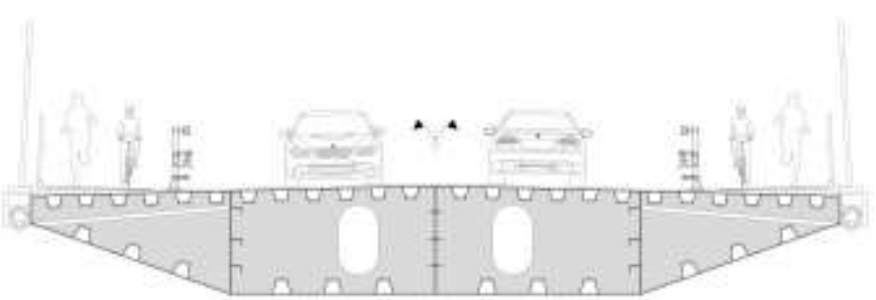

Figure 2. The bridge deck

\section{B. Parametric excitation}

As known, the usual approach to the study of parametric excitation consists of an evaluation of the cable resonance condition from dynamic equilibrium equations of a single cable under harmonic motion of its supports.

The response to a harmonic of frequency $\omega$ is not increased exclusively at resonance (when the fundamental frequency of cable $\left.\omega_{1}=\omega\right)$, but also at specific ratios $\beta=\omega / \omega_{1}=2 / \delta=1 / r=\{1 / 2 ; 1 / 3 ; 2 ; 3\}[1]$.

Considering the first parametric resonance in correspondence of a subharmonic of order $1 / 2\left(\delta^{2}=1, r=1 / 2\right.$, $\beta=2)$, the threshold amplitude for occurrence parametric excitation is given by

$$
x_{\mathrm{B}, \mathrm{lim}}=4 \xi_{1} X_{0}
$$

where $X_{0}=T L /\left(E A_{0}\right)$ is the elastic elongation of the cable [1]. The threshold amplitude of oscillation has been calculated for bridge stays considering three possible damping coefficients: $0.2 \%, 0.5 \%$ and $1.0 \%$ (Fig. 3 ). These values are lower for stiffer stays (\#4 to \#7).

Fig. 4 shows amplitudes of steady-state oscillation $\alpha\left(x_{\mathrm{B}}\right)$

$$
a\left(x_{\mathrm{B}}\right)=\frac{4}{\pi} \sqrt{\frac{X_{0} L}{3}} \sqrt{1-\delta^{2}+\sqrt{\delta^{4}\left(\frac{x_{\mathrm{B}}}{2 X_{0}}\right)^{2}-4 \delta^{2} \xi_{1}^{2}}}
$$

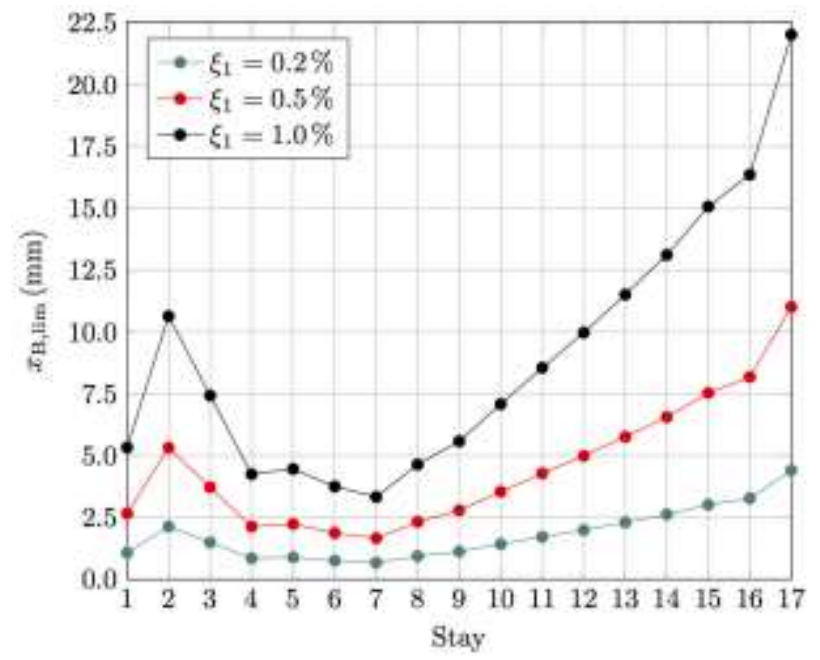

Figure 3. Threshold amplitude of parametric oscillation for $\delta=1$.

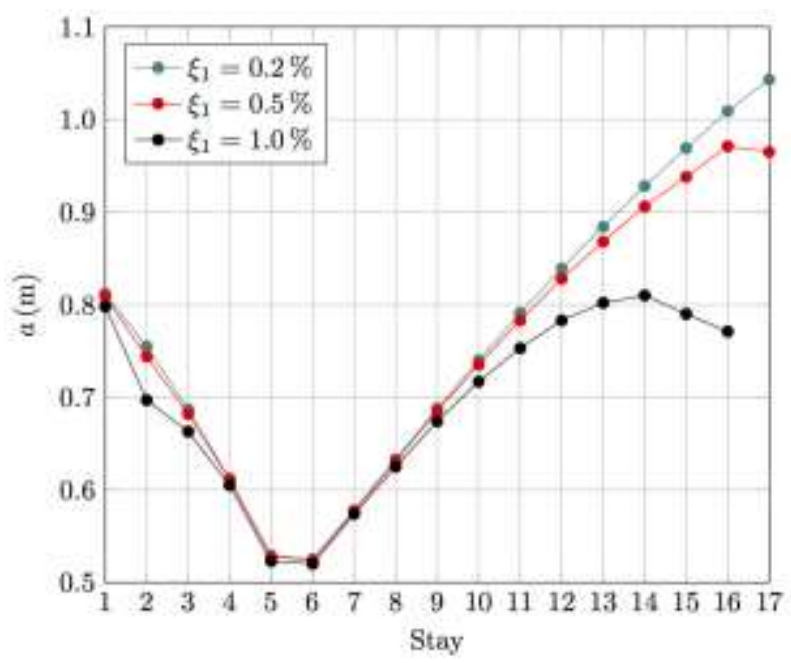

Figure 4. Amplitudes of steady-state parametric oscillation for $x_{\mathrm{B}}=20 \mathrm{~mm}$

for a sinusoidal excitation of $20 \mathrm{~mm}$ amplitude, at twice the cable frequency $(\delta=1)[1]$.

A damping ratio of $1.0 \%$ affects only the behavior of weaker stays. In general, damping is important only to 
prevent parametric excitation: once oscillation sets up the amplitude is almost damping-independent [1], as clearly shown in Fig. 5 for stay \#7.

\section{External excitation}

External excitation induces amplitudes of vibration almost halving those induced by parametric excitation, which are therefore considered the most important [1]. This aspect appears clearly comparing diagram in Fig. 6, referring to amplitude of steady state external excitation of stay \#7, with the previously discussed Fig. 5.

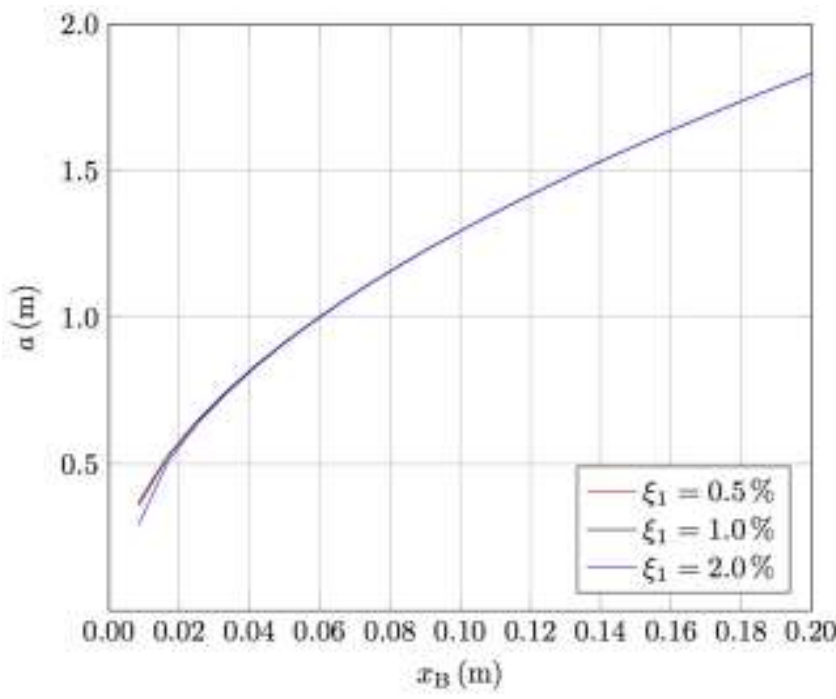

Figure 5. Amplitudes of steady-state parametric oscillation for stay \#7.

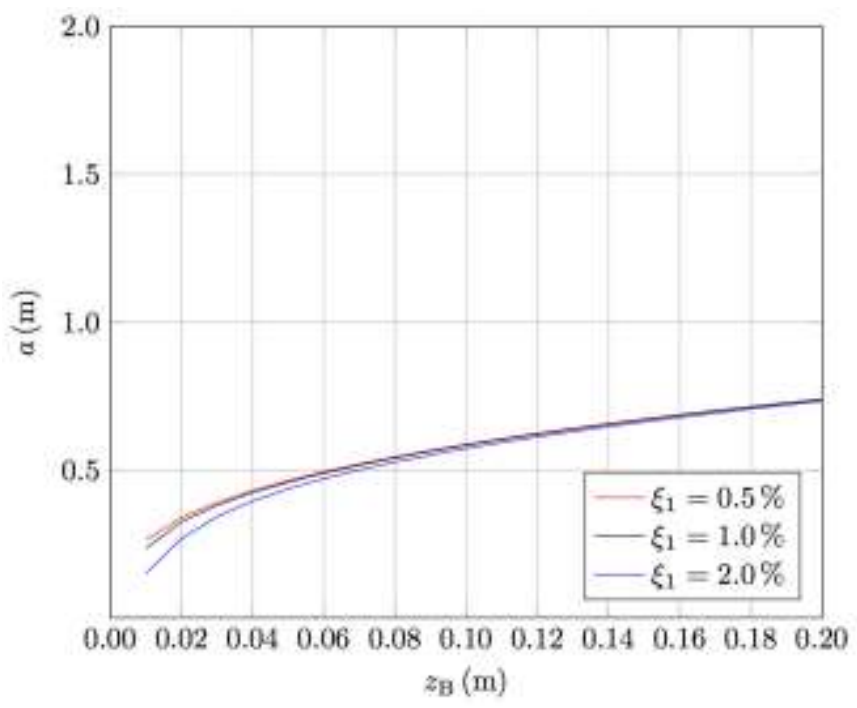

Figure 6. Amplitudes of steady-state external oscillation for stay \#7.

Amplitudes of steady-state external oscillation for stay \#7 have been calculated through the solution developed by Nayfeh and Mook in the vicinity of primary resonance $(r \approx 1)[7]:$

$$
a\left(z_{\mathrm{B}}\right)=\frac{z_{\mathrm{B}}}{\pi \xi_{1}} C \sin \gamma
$$

$$
C=\frac{1-\frac{\lambda^{2}}{\pi^{2}}}{\sqrt{1+\frac{1}{2}\left(\frac{2}{\pi}\right)^{4} \lambda^{2}}}
$$

The phase of response $\gamma$ is obtained by numerical solution of

$$
\sin ^{2} \gamma \tan \gamma=\frac{32}{3} X_{0} L \frac{\left[1+\frac{1}{2}\left(\frac{2}{\pi}\right)^{4} \lambda^{2}\right]^{4}}{\left[1-\frac{\lambda^{2}}{\pi^{2}}\right]\left[1-32 \frac{\lambda^{2}}{\pi^{4}}\right]} \frac{\xi_{1}^{3}}{z_{\mathrm{B}}^{2}} .
$$

\section{Cable-structure interaction}

Cable-structure interaction can be studied through the ratio $r$ of fundamental cable frequency $f_{1 \mathrm{c}}$ to bridge global frequency $f_{\mathrm{b}}$. The value $r \approx 1$ defines the region where global modes may provide external excitation. Subharmonic $(r \approx 0.5)$ and superharmonic $(r \approx 2)$ resonance conditions bound two nonlinear interaction regions, where global modes may provide parametric and angle variation excitation of local modes respectively [8,9]. The excitation from angle variation between cable tension and bridge girder is a phenomenon detailed by Gattulli and Lepidi and Gattulli et al. $[9,10]$.

Fig. 7 and Fig. 8 show values of $r$ considering each cable frequency (Table I), ten first vertical global modes and first torsional mode (Table II). It emerges that: stays from \#3 to \#11, \#16 and \#17 are vulnerable to external excitation of six first vertical global modes; all stays are vulnerable to parametric excitation of higher global modes; and stays \#2 and \#9 are vulnerable to angle variation excitation.

TABLE II. BRIDGE GLOBAL FREQUENCIES.

\begin{tabular}{rcl}
\hline Mode & $f_{\mathrm{b}}(\mathrm{Hz})$ & Description \\
\hline 1 & 0.543 & $1^{\circ}$ vert. (V1) \\
2 & 0.870 & $1^{\circ}$ lat. (L1) \\
3 & 0.913 & $2^{\circ}$ lat. (L2) \\
4 & 0.946 & $2^{\circ}$ vert. (V2) \\
5 & 1.311 & $3^{\circ}$ vert. (V3) \\
6 & 1.556 & $4^{\circ}$ vert. (V4) \\
7 & 1.921 & $5^{\circ}$ vert. (V5) \\
8 & 1.923 & $1^{\circ}$ tors. (T1) \\
9 & 1.993 & $3^{\circ}$ lat. (L3) \\
10 & 2.107 & $6^{\circ}$ vert. (V6) \\
11 & 2.820 & $7^{\circ}$ vert. (V7) \\
12 & 2.892 & $4^{\circ}$ lat. (L4) \\
13 & 2.995 & $8^{\circ}$ vert.(V8) \\
14 & 4.142 & $9^{\circ}$ vert. (V9) \\
15 & 4.678 & $5^{\circ}$ lat. (L5) \\
16 & 4.809 & $1^{\circ}$ vert. (V10) \\
17 & 5.179 & $1^{\circ}$ long. (Lo1) \\
18 & 5.284 & $2^{\circ}$ tors. (T2) \\
19 & 5.474 & $1^{\circ}$ vert. (V11) \\
20 & 5.786 & $6^{\circ}$ lat. (L6) \\
\hline
\end{tabular}




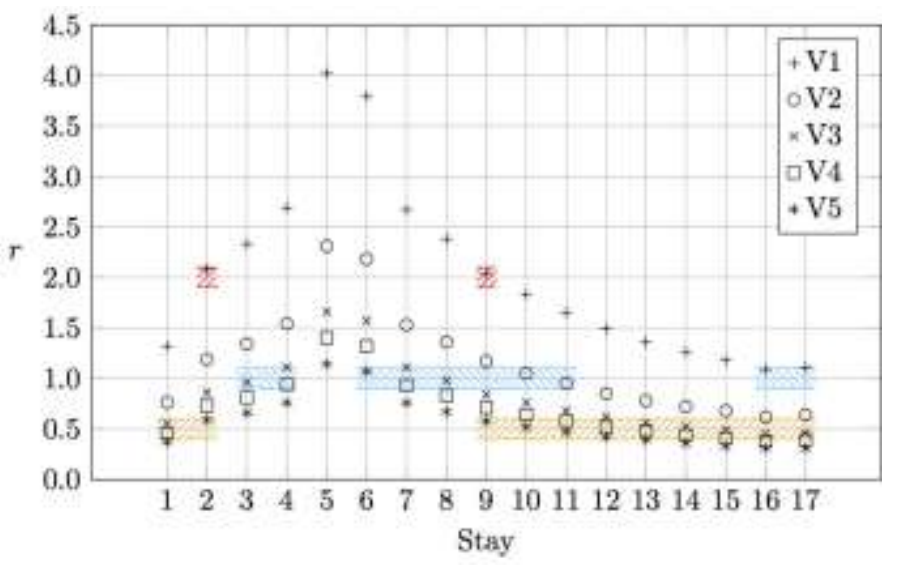

Figure 7. Ratio $r=f_{1 \mathrm{c}} / f_{\text {bridge }}$ for modes V1-V5.

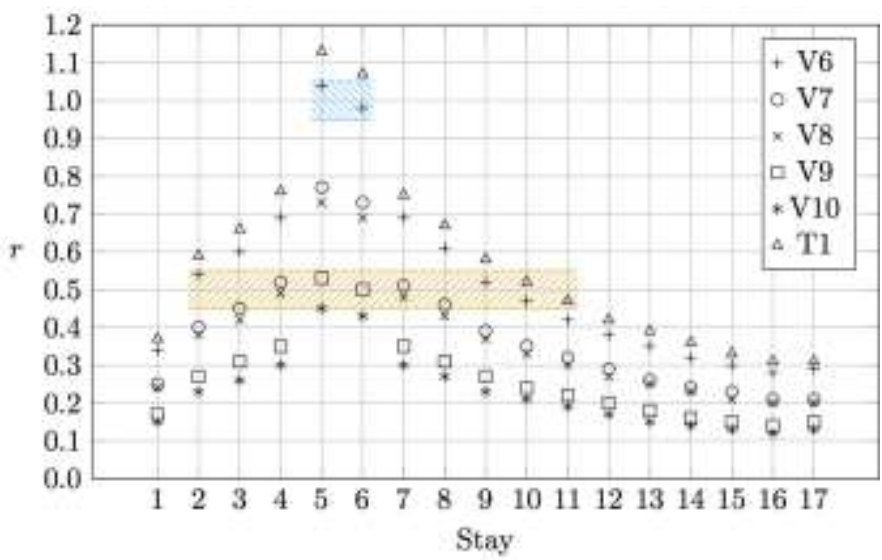

Figure 8. Ratio $r=f_{1 \mathrm{c}} / f_{\text {bridge }}$ for modes V6-V10 and T1.

\section{v. Damper design}

As already anticipated, mounting viscous dampers close to deck anchorages is an efficient way to control all kind of cable vibrations. Moreover this is the least expensive technique in retrofitting existing bridge suffering from wind/rain-wind induced vibrations. Therefore, the evaluation of damping needed is crucial in preventing large cable vibrations.

For all stays a damping ratio $\xi_{1}>0.3 \%$ is generally recommended as appropriate to control dry inclined galloping and rain wind induced vibrations [4].

However for cable stays longer than $80 \mathrm{~m}$ provisions should be made for the installation of dampers to ensure that critical damping ratios exceed $0.5 \%$ in the first mode [11].

Focusing on the longest stay (\#17, L=206 m), neglecting intrinsic structural damping and taking account of all vibration modes in the range $(0.3 \div 3) \mathrm{Hz}$ [1], a damping ratio $\xi>0.3 \%$ is requested for the first five modes:

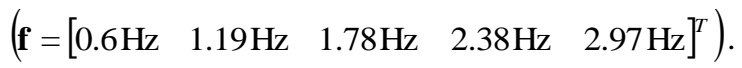

The simplified formula derived by Fujino and Hoang $[1,12]$ was used in the design of an optimal passive damper:

$$
\begin{gathered}
\frac{\xi_{n}}{x_{\mathrm{c}} / L}=R_{n} R_{E J} R_{k \mathrm{EJ}} \frac{\eta_{n} \eta_{k \mathrm{EJ}}}{1+\left(\eta_{n} \eta_{k \mathrm{EJ}}\right)^{2}} \\
R_{n}=\left\{\begin{array}{c}
\frac{1}{\left[\tan \left(\frac{k_{n} \pi}{2}\right)-\left(\frac{k_{n} \pi}{2} \frac{x_{\mathrm{c}}}{L}\right)\right]^{2}} \text { if } n \text { is even, } \\
\tan ^{2}\left(\frac{k_{n} \pi}{2}\right)+\frac{12}{\lambda^{2}}\left(\frac{k_{n} \pi}{2}\right)^{2}
\end{array} \text { if } n\right. \text { is odd. } \\
R_{E J} \approx \frac{(1-q)^{2}}{1-q-0.5 r q^{2}} \\
q=\frac{1-\exp (-r)}{r} \\
R_{k \mathrm{EJ}}=\frac{\frac{x_{\mathrm{c}}}{L}}{1+\overline{\eta_{k}} \eta_{\mathrm{EJ}}}
\end{gathered}
$$

where

$\zeta$ is a bending stiffness coefficient appearing in the Mehrabi and Tabatabai formula;

$x_{\mathrm{c}}$ is the distance of the damper from the cable anchorage;

$\eta_{n}=\eta k_{n} \pi x_{c} / L$ is a non-dimensional damping parameter;

$k_{n}=\omega_{n} / \omega_{01}=f_{n} / f_{01}$ is the ratio of $n$-th cable frequency to the fundamental frequency of corresponding taut

$$
\text { string }\left(\omega_{01}=\frac{\pi}{L} \sqrt{\frac{T}{m}}\right) \text {; }
$$

$R_{n}$ is a reduction factor due to sag effect;

$R_{\mathrm{EJ}}$ is a reduction factor due to bending effect;

$\eta_{k \mathrm{EJ}}=\eta_{\mathrm{EJ}}+1 / \overline{\eta_{k}}$ is a non-dimensional damping parameter related to the stiffness $k$ of support and to the bending stiffness of damper;

$\eta_{\mathrm{EJ}}=1-q-0.5 r q^{2} \quad$ is a non-dimensional damping parameter related to the bending stiffness of the damper;

$\overline{\eta_{k}}=\left(k x_{c}\right) / T$ is a non-dimensional damping parameter related to the stiffness $k$ of support;

$R_{k \mathrm{EJ}}$ is a reduction factor associated with the support stiffness.

Assuming that viscous damper is linear, the maximum modal damping ratio is obtained by

$$
\frac{\xi_{n, \max }}{x_{\mathrm{c}} / L}=0.5 R_{n} R_{E J} R_{k \mathrm{EJ}}
$$

and occurs for 


$$
\eta_{n, \mathrm{opt}}=\frac{1}{\eta_{k \mathrm{EJ}} k_{n}} .
$$

With damper inserted in a deviation guide of height $\mathrm{h}=2.5 \mathrm{~m}$ and an inclination of cable chord $\theta=25^{\circ}$, $x_{\mathrm{c}}=h / \sin \theta=6 \mathrm{~m}$ and $x_{\mathrm{c}} / L=0.03$. For stay $\# 17 \zeta=609$, so $R_{\mathrm{EJ}}=0.97$ and

$$
\begin{gathered}
\mathbf{k}=\left[\begin{array}{lllll}
1.020 & 2.007 & 3.010 & 4.013 & 5.017
\end{array}\right]^{T} \\
\mathbf{R}_{\mathbf{n}}=\left[\begin{array}{lllll}
0.94 & 1.00 & 0.87 & 1.00 & 0.45
\end{array}\right]^{T} .
\end{gathered}
$$

Neglecting support stiffness, the achievable maximum modal damping ratio is

$$
\xi_{\max }=\left[\begin{array}{lllll}
1.313 \% & 1.397 \% & 1.215 \% & 1.397 \% & 0.629 \%
\end{array}\right]^{T}
$$

occurring for

$$
\boldsymbol{\eta}_{\text {opt }}=\left[\begin{array}{lllll}
1.07 & 0.54 & 0.36 & 0.27 & 0.22
\end{array}\right]^{T} .
$$

Setting $\xi_{5}=0.3$ in (6) and solving for $\eta$, it results $\eta=0.61$. Knowing that

$$
\eta=\frac{\pi c}{m L \omega_{01}}
$$

and that for stay \#17 $\omega_{01}=3.54 \mathrm{rad} / \mathrm{s}$, the requested damper size is

$$
c=59627 \mathrm{Ns} / \mathrm{m} \approx 60 \mathrm{kNs} / \mathrm{m} \text {. }
$$

Finally, referring to the so-called universal curve reported in Fig. 9, the abscissas

$$
x_{n}=\frac{\eta n \pi x_{\mathrm{c}} / L}{\pi^{2}}
$$

can be evaluated,

$$
\mathbf{x}=\left[\begin{array}{lllll}
0.88 & 1.72 & 2.58 & 3.44 & 4.31
\end{array}\right]^{T},
$$

so obtaining the modal damping ratios

$$
\xi=\left[\begin{array}{lllll}
1.28 \% & 1.26 \% & 0.87 \% & 0.81 \% & 0.30 \%
\end{array}\right]^{T},
$$

which demonstrate that a critical damping ratio $\xi_{1}=1.28 \%$ is sufficient to control all kind of cable vibrations of stay \#17.

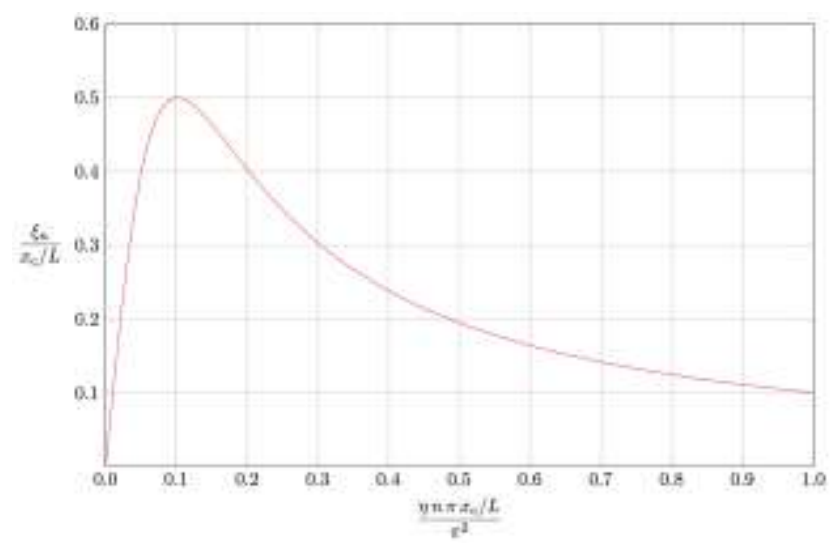

Figure 9. Universal curve relating modal damping ratio $\xi_{n}$ with damper size $c$, location of damper $x_{\mathrm{c}}$ and cable parameters $m, L$ and $\omega_{01}[1]$.

\section{vI. Concluding remarks}

The dynamic stability of stays under indirect excitation has been investigated for a relevant case study. In particular, parametric excitation, external excitation and cable-structure interaction have been considered.

The results confirm that damping is very significant to prevent parametric excitation, while external excitation is less important because it induces smaller amplitudes of vibration. Moreover, cable-structure interaction is a relevant matter for the considered bridge. At the end, a viscous damper has been designed as possible countermeasure for the improvement of dynamic stability of stays.

\section{References}

[1] E. de Sá Caetano, Cable Vibrations in Cable-Stayed Bridges. IABSEAIPC-IVBH, 2007.

[2] R. Castelluccio, "Il nuovo ponte strallato sull'Arno a Figline Valdarno," Università $\quad$ di https://etd.adm.unipi.it/theses/available/etd-09082016-181846/, 2016.

[3] A. Mehrabi and H. Tabatabai, "Unified finite difference formulation for free vibration of cables," Journal of Structural Engineering, vol. 124, no. 11, pp. 1313-1322, 1998.

[4] P. Croce, P. Formichi, F. Landi and R. Castelluccio, "Assessing wind induced stay vibrations in bridges", International Conference on Advances in Civil, Structural and Mechanical Engineering, Zurich, 2017.

[5] H. M. Irvine, "Free Vibrations of Inclined Cables," Journal of the Structural Division, vol. 104, no. 2, pp. 343-347, 1978.

[6] P. Croce, "Non-linear behavior of heavy stays," International Journal of Solids and Structures, vol. 50, no. 7, pp. 1093-1107, Apr. 2013.

[7] A. H. Nayfeh and D. T. Mook, Nonlinear Oscillations. John Wiley \& Sons, 1979.

[8] E. de Sá Caetano, A. Cunha, V. Gattulli, and M. Lepidi, "Cable-deck dynamic interactions at the International Guadiana Bridge: On-site measurements and finite element modelling," Structural Control and Health Monitoring, vol. 15, no. 3, pp. 237-264, 2008.

[9] V. Gattulli and M. Lepidi, "Nonlinear interactions in the planar dynamics of cable-stayed beam," International Journal of Solids and Structures, vol. 40, no. 18, pp. 4729-4748, 2003.

[10] V. Gattulli, M. Lepidi, J. H. G. Macdonald, and C. A. Taylor, "Oneto-two global-local interaction in a cable-stayed beam observed through analytical, finite element and experimental models," International Journal of Non-Linear Mechanics, vol. 40, no. 4, pp. 571-588, 2005.

[11] "Eurocode 3: Design of steel structures - Part 1-11: Design of structures with tension components." 2010.

[12] Y. Fujino and N. Hoang, "Design Formulas for Damping of a Stay Cable with a Damper," Journal of Structural Engineering, vol. 134, no. 2, pp. 269-278, Feb. 2008. 